\title{
Transgenerational epigenetic inheritance in animals
}

\section{Igor Kovalchuk*}

Department of Biological Sciences, University of Lethbridge, Lethbridge, AB, Canada

*Correspondence: igor.kovalchuk@uleth.ca

Organisms are in a constant interaction with abiotic and biotic components of their surroundings. Whereas many and perhaps most of the encounters pass with transient changes at the molecular and metabolic levels, some have a profound impact on the phenotypic appearance of an organism and, more importantly, on the phenotypic appearance of their progeny. Since genetic changes are rare and random in nature, their influence on species or/and population evolution, especially in higher eukaryotes, may not be as dramatic and important as it was believed to be. In contrast, environmentally induced transgenerational phenotypic changes, that is what epigenetics in its narrow sense is, are more likely to have an appreciable effect on stress adaptation and the process of microevolution.

To date, there are many more definitions of epigenetics and transgenerational responses. Whether or not one appreciates a topic of transgenerational epigenetic responses and their heritable nature, there is still a substantial debate about the nature of these and other related phenomena. The current opinion article attempts to present several different views on transgenerational epigenetic inheritance in animals, including various definitions of this and other related terms. It proposes to standardize the meaning behind these definitions and suggests some minimum requirements for reporting transgenerational effects and transgenerational heritable effects of stress.

First of all, what is epigenetics? There is substantial disagreement even over a simple (or not that simple) definition of the term epigenetics itself. The actual term "epigenetics" was first used by Conrad Hal Waddington, and in its strict meaning, it was supposed to describe the interaction between organisms and their environment that resulted in the appearance of a particular phenotype. Indeed, it is impossible nowadays to talk about epigenetics without considering its critical component - the interaction with environment. Also, since the emergence of epigenetics has been associated with the concept of "soft inheritance," heritability of changes may be one of the requirements for the definition of epigenetics.

One of the modern definitions of epigenetics suggests that epigenetics represents heritable changes in gene expression that do not involve changes in the genetic code. Again, the word "heritable" is there, which implies that changes must be observed in the progeny of an organism. Nowadays, epigenetic mechanisms include a variety of modifications such as DNA methylations, a vast plethora of histone modifications, and replacements of regular histones with histone variants, changes in chromatin structure as a consequence of differential binding of non-histone proteins, and, finally, the activity of various small noncoding RNAs. It is debatable whether the regulation of gene expression through small RNAs should be considered to be part of epigenetic regulation. There seems to be no clear position on that. In my interactions with many reviewers and editors encountered in the processes of submission/review to many journals, I noticed that the majority of scientists believe that the regulation of gene expression via small RNAs is part of epigenetic regulation.

As summarized by Ho and Burggren (2010), the definition of epigenetics largely depends on whether the term is used by a molecular biologist, a geneticist, a developmental biologist, or an animal physiologist. The same is true for cell or epigenetic memory. The term transgenerational inheritance or heritability reflects changes at the cellular level (mitotic and meiotic), changes in population levels, or even behavioral level changes (Ho and Burggren, 2010). For further details of these definitions, I refer the reader to this review and the list of works cited.

If we adhere to the opinion that a more narrow definition of epigenetics involves heritability of changes, we need to consider several other related definitions such as a transgenerational response, a transgenerational transfer, and transgenerational memory, all of which are believed to occur without changes in the genetic makeup. In the past, these more restricted terms that define epigenetics were referred to as "parental transfer," "genomic imprinting," and more recently "transgenerational memory" and "transgenerational response," or "transgenerational plasticity" (reviewed in Ho and Burggren, 2010). Most of these terms emerged in an attempt to describe changes in the progeny of an organism exposed to stress. Frequently but not always, the progeny of stressed organisms acquired some beneficial traits such as the ability to respond to stress. Thus, transgenerational response can be defined as changes in physiology in response to stress of the progeny whose one parent was exposed to prior to fertilization.

Are transgenerational effects always heritable? Although the transgenerational transfer or transgenerational memory may include many different factors that can be attributed to epigenetics, such as the pool of differentially expressed small RNAs either in the cytoplasm of the ovum or in the nucleus of both the ovum and sperm cells, some of the factors are not necessarily epigenetic in nature. For example, the accumulation of proteins and metabolites in the cytoplasm and organelles of maternal gametes in response to stress may significantly alter the development and phenotypic appearance of an organism. Such events are definitely part of a transgenerational response but are hardly epigenetic in nature. A broad definition of the transgenerational transfer implies the transfer of the phenotypic appearance into the progeny independently of genetic factors. In contrast, transgenerational epigenetic inheritance is the transfer of the phenotypic appearance as a result of the transfer of epigenetic marks. These epigenetic modifications are assumed to be solely responsible for phenotypic changes.

Thus, heritability of transgenerational events may rely on, among other epigenetic events, DNA methylation, and histone 
modifications. Since DNA methylation and histone modifications undergo two reprogramming events during the formation of gametes and shortly after fertilization, the inheritance of modifications that occurs in parental gametes requires escaping reprogramming, not only once while passing changes from F0 to F1 but also twice while passing them further to F2. Therefore, depending on the timing of exposure to stress during the development of an organism, the real nature of heritability of transgenerational responses can be questioned. Since in animals, gametes are formed during early development, the appearance of a new phenotype in the immediate progeny (F1) due to some epigenetic changes may not be an inheritance event because gametes of a F0 individual will be exposed to the said stress and thus fix epigenetic changes upon fertilization. Still there will have to be at least one event of escaping reprogramming after fertilization. New epigenetic modifications in the gametes of the exposed F0 individual and their persistence through the fertilization event as well as their propagation to somatic and gametic tissues of the F1 progeny associated with the appearance of a new phenotype would be a serious proof of transgenerational epigenetic inheritance. However, to stick to more conservative views, one needs to observe the phenotype and associated epigenetic changes in the F2. If presumably stress exposure has occurred during pregnancy, then technically both F1 (an embryo) and F2 (its gametes) progenies have a chance to experience stress exposure.

Again, as in the previous case, epigenetic changes caused by stress exposure would have to escape at least one round of reprogramming. In this case, the observation of phenotypic changes and associated epigenetic modifications in the F3 progeny is required to claim that the phenomenon of heritable transgenerational response is real. Thus, from a conservative point of view, heritable responses may be considered to be heritable changes in gene expression in mammals that occur in the F3 progeny of a stressed organism.

Do all stresses equally cause a transgenerational response? Does the developmental stage of an organism play a major role in this response? How frequent should exposure occur to cause a short-term (epigenetic) or long-term (genetic) response of an organ- ism? Most of these questions either are not answered at all or do not have a clear answer yet. Parsons suggests that exposure to unfamiliar/unusual stresses and exposure to a high level/intensity of stress are less likely to cause an adaptive evolutionary response as compared to exposure to familiar stresses of moderate levels/intensities (Parsons, 1994). It can be hypothesized that slight fluctuations in the environment are dealt with at the molecular level, and they do not substantially change homeostasis and therefore do not result in traceable changes in genetic and epigenetic inheritance. Moderate environmental exposures may lead to an epigenetic response of an organism, and epigenetic memory of this response can be passed on to the next generations of somatic cells as well as to progeny. At the same time, according to Parsons (1994), harsh stresses are unlikely to trigger an adaptive response but may lead to "evolutionary stasis." Extreme fluctuations in the environment are associated with the extreme energy consumption requirements of organisms to achieve stress tolerance. Since such responses are typically associated with the slower metabolism and stronger regulatory systems, they are more likely to cause evolutionary stasis. Similarly, a response to general non-specific stress is also associated with enhanced homeostasis that leads to decreased metabolism and therefore, reduced fitness (reviewed in Badyaev, 2005).

Most of the epigenetic changes are transient in nature, and although they are heritable, they are unlikely to be inherited beyond a couple of generations. Such short-term changes in response to an environmental stimulus commonly occur if the frequency of stimulus encounters is greater than the generation time of an organism but shorter than is necessary to permanently fix changes in the genetic code. In contrast, it is plausible to speculate that the more frequent and long lasting encounters of stress, such as habitat changes, may trigger a long-term response in the form of permanent genetic changes. Individuals who experience such changes may no longer require a short-term response to the environment. It is even more possible to suggest that a short-term response (microevolution, in a way) frequently precedes a long-term response. It can be assumed that changes in DNA methylation or histone modifications associated with a specific chromatin structure are transformed into genetic changes. It is commonly believed that changes in DNA methylation are associated with altered frequencies of genetic changes, whereas hypomethylation is associated with the more frequent genomic rearrangements, and hypermethylation - with more frequent $\mathrm{C} \rightarrow \mathrm{T}$ point mutations at methylated cytosine. A similar situation may occur with permissive or restrictive chromatin marks such as histone acetylation/methylation, etc., wherein chromatin that is more loose becomes more permissive for genomic rearrangements. However, it remains to be shown experimentally that stress-induced epigenetic changes that cause the appearance of a particular phenotype result in genetic changes that maintain or perhaps amplify such phenotype.

No matter what experimental model is chosen and what types of stress and stress regimes are studied, caution needs to be exercised before claiming a heritable transgenerational response. As mentioned above, the occurrence of epigenetic changes, whether it is DNA methylation, histone modifications, or altered small RNA profiles, needs to be demonstrated, and its association with a particular phenotype should be apparent. The persistence of the phenotype (and an epigenetic mark) in the F2 generation (F3 in case of exposure of during pregnancy) needs to demonstrated.

Finally, I apologize for not having cited many excellent original research papers and reviews on the topic as this was not the purpose of the current opinion article.

\section{REFERENCES}

Badyaev, A. V. (2005). Stress-induced variation in evolution: from behavioural plasticity to genetic assimilation. Proc. Biol. Sci. 272, 877-886.

Ho, D. H., and Burggren, W. W. (2010). Epigenetics and transgenerational transfer: a physiological perspective. J. Exp. Biol. 213, 3-16.

Parsons, P. A. (1994). Morphological stasis: an energetic and ecological perspective incorporating stress. $J$ Theor. Biol. 171, 409-414.

Received: 24 February 2012; accepted: 22 April 2012; published online: 09 May 2012.

Citation: KovalchukI (2012) Transgenerational epigenetic inheritance in animals. Front. Gene. 3:76. doi: 10.3389/ fgene.2012.00076

This article was submitted to Frontiers in Epigenomics, a specialty of Frontiers in Genetics.

Copyright (C) 2012 Kovalchuk. This is an open-access article distributed under the terms of the Creative Commons Attribution Non Commercial License, which permits non-commercial use, distribution, and reproduction in other forums, provided the original authors and source are credited. 